\title{
EFFECT OF THE CHROMOPHORES STRUCTURES ON THE PERFORMANCE OF SOLID-STATE DYE SENSITIZED SOLAR CELLS
}

\author{
HAINING TIAN*,**, ANDREA SOTO*, BO XU*, LICHENG SUN*,, \\ ANDERS HAGFELDT ${ }^{\Uparrow}$, FRANCISCO FABREGAT-SANTIAGO ${ }^{\dagger}$, IVAN MORA-SERO ${ }^{\dagger}$, \\ YONG SOO KANG", JUAN BISQUERT ${ }^{\dagger, \dagger \dagger}$ and EVA M. BAREA ${ }^{\dagger,+}$ \\ *Department of Chemistry, Organic Chemistry \\ KTH Royal Institute of Technology, S-100 44 Stockholm, Sweden \\ ${ }^{\dagger}$ Photovoltaic and Optoelectronic Devices Group \\ Physics Department, Universitat Jaume I. 12071 Castelló, Spain \\ *Centro de Investigación en Electroquímica y Energía Química (CELEQ) \\ Universidad de Costa Rica \\ Ciudad de la Investigación, Apartado 115012060, \\ San José, Costa Rica \\ ${ }^{\S}$ State Key Laboratory of Fine Chemicals \\ DUT-KTH Joint Research Center on Molecular Devices \\ Dalian University of Technology (DUT), 116024 Dalian, P. R. China \\ "Department of Chemistry-Ångström Laboratory \\ Uppsala University, 75105 Uppsala, Sweden \\ "Energy Materials Laboratory, Department of Chemical Engineering \\ Hanyang University, Seoul 133-791, South Korea \\ **hainingt@kth.se \\ †'bisquert@uji.es \\ Hbarea@uji.es
}

Received 23 February 2014

Accepted 26 March 2014

Published 6 May 2014

The effect of metal-free chromophores on dye-sensitized solar cell performance is investigated. Solid state dye-sensitized solar cells (ssDSCs) using different molecular sensitizers based on triphenylamine (TPA) with thiophene linkers and different alkyl chain in the donor unit have been characterized using impedance spectroscopy (IS). We show that different molecular structures play a fundamental role on solar cell performance, by the effect produced on $\mathrm{TiO}_{2}$ conduction band position and in the recombination rate. Dye structure and its electronic properties are the main factors that control the recombination, the capacitance and the efficiency of the cells. A clear trend between the performance of the cell and the optimization level of the blocking effect of

\$Corresponding author. 
the dye structure has been identified in the solid state solar cells with Spiro-OMeTAD hole conductor.

Keywords: Dye-sensitized solar cells; solid state; metal-free chromophores; impedance spectroscopy; Spiro-OMeTAD.

\section{Introduction}

Solid state dye-sensitized solar cells (ssDSCs) have attracted wide interest from scientists since the first report by Bach and co-authors in $1998 .{ }^{1}$ In this kind of light-to-electricity conversion device, the photosensitizer undertakes the light harvesting and electron injection to semiconductor, and it plays an important role in the final efficiency in the device. Organic dyes, ${ }^{2-6}$ metal complexes, ${ }^{7,8}$ quantum $\operatorname{dots}^{9-12}$ as well as perovskite ${ }^{13-18}$ have been employed as the light absorber in ssDSCs or "meso-superstructured" solar cell (MSSC). As one kind of the photosensitizer, organic dyes are investigated intensely due to low cost, easy synthesis and tunable light absorption properties. In 2010, Cai and co-workers reported an efficiency over $6 \%$ using an organic sensitizer C220 and (N,N-di-p-methoxyphenyl-amine)-9,9'-spiro-bifluorene (Spiro-OMeTAD) as hole transport material (HTM). ${ }^{3}$ Recently, an impressive efficiency of $7.2 \%$ was achieved with the combination of tris $(2-(1 \mathrm{H}-$ pyrazol-1-yl)pyridine)cobalt(III) doped Spiro-OMeTAD
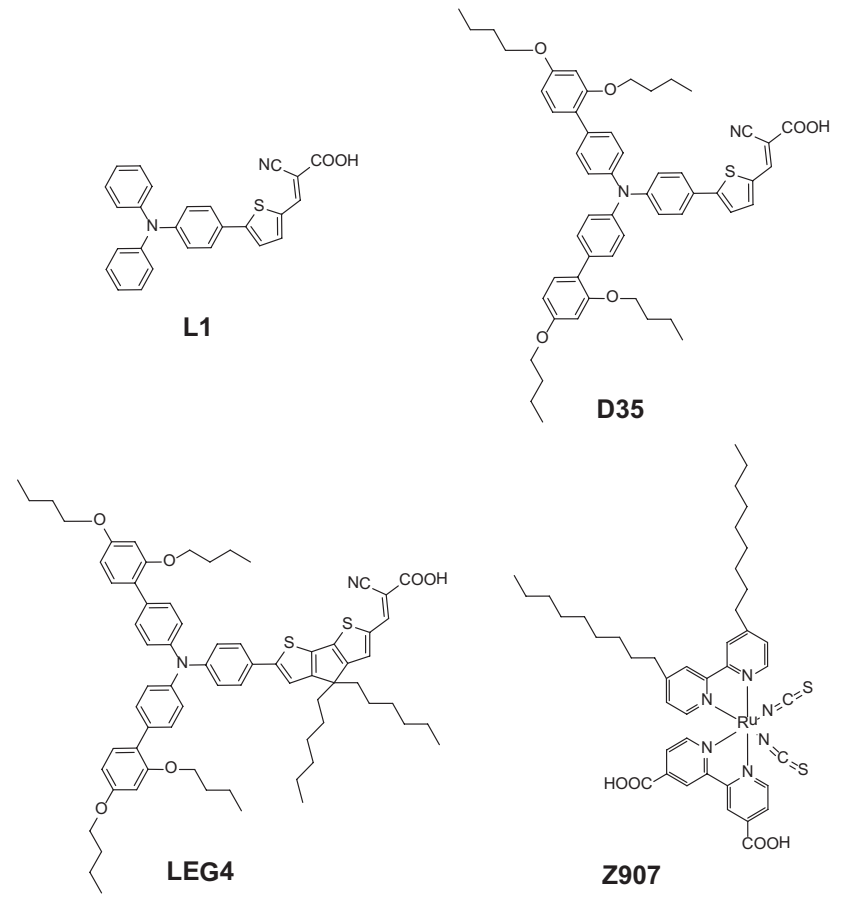

Fig. 1. The molecular structures of the selected dyes. and organic photosensitizer Y123. ${ }^{19}$ From these previous studies, the optimization of dye structure can greatly improve the performance of ssDSCs.

In this work, four dyes with differences in its molecular skeleton are selected to study the effect of dye structures on the performance of ssDSCs. All the devices are characterized with impedance spectroscopy (IS) in order to determine all the processes involved in the solar cell performance, with the aim to determine the relation between solid cell performance and dye structure, obtaining an excellent understanding about which dye structure is more suitable to use to fabricate high efficiency ssDSCs. The dyes are shown Fig. 1. L1, D35, LEG4 are organic dyes and Z907 is a Ru-based photosensitizer employed as reference dye. L1 has the most simple dye structure among these dyes, bearing triphenylamine (TPA) as donor, thiophene as linker and cyanoacrylic acid as anchoring group. Comparing with L1, at D35 dye introduced more alkyl chain in donor unit. Using cyclopenta-[1,2-b:5,4-b']-dithiophene (CPDT) instead of thiophene in D35 forms LEG4 dye.

\section{Experimental Section}

\subsection{Dye characterization}

L1, D35 and LEG4 were provided by Dyenamo Company. Z907 was purchased from Solaronix Company. All other chemicals and solvents were purchased from Sigma-Aldrich and used as received. Figure 2 shows the absorption spectra of different dyes and Fig. 3 depicts the energy levels of components involved in ssDSCs device. UV-Vis absorbance spectra were recorded on a Lambda $750 \mathrm{UV}$-Vis spectrophotometer. The Highest Occupied Molecular Orbital (HOMO) of L1 was tested by cyclic voltammetry (CV) method. Lowest Unoccupied Molecular Orbital (LUMO) level was calculated by HOMO$E_{0-0}$, where $E_{0-0}$ was obtained by the wavelength $(\lambda)$ at intersection of absorption and fluorescence spectra, $E_{0-0}=1240 / \lambda$. The HOMO and LUMO data of D35, LEG4 and Z907 were taken from literatures. ${ }^{20,21}$ 


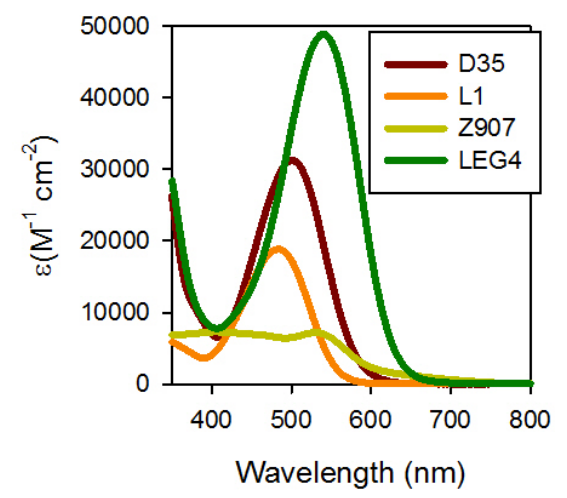

Fig. 2. UV-Vis spectra of LEG4, D35, L1 and Z907 dyes.

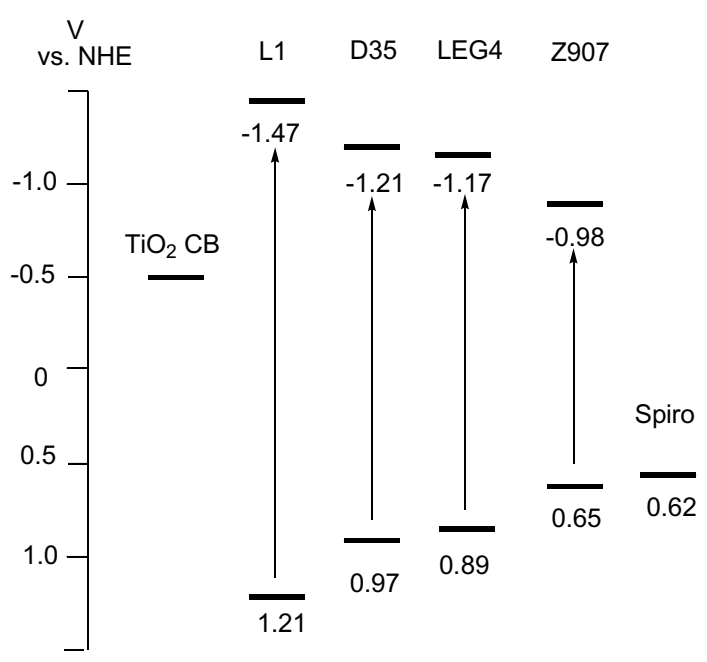

Fig. 3. The diagram of energy levels of the components in ssDSCs devices.

The corresponding physical properties of dyes are collected in Table 1. For organic dyes, introducing butoxyl phenyl units in donor or more conjugated segment in linker can make the dye's absorption spectrum red-shifted as well as increase dye's extinction coefficient $(\varepsilon)$. One can find that LEG4 has the highest $\varepsilon$ value of $49000 \mathrm{M}^{-1} \cdot \mathrm{cm}^{-2}$ among these dyes at its maximum absorption peak $540 \mathrm{~nm}$. Z907 dye shows the lowest $\varepsilon$ value of $7100 \mathrm{M}^{-1} \cdot \mathrm{cm}^{-2}$ among these dyes at $531 \mathrm{~nm}$. Theoretically, the dye with higher $\varepsilon$ is able to harvest more light in ssDSCs devices, because the solid devices usually employ thin $\mathrm{TiO}_{2}$ film in order to increase the penetration of HTM, such as Spiro-OMeTAD, and reduce the total electron recombination.

From the diagram of energy levels of different dyes in Fig. 3, we observe that all dyes have LUMO levels that are more negative than conduction band (CB) of $\mathrm{TiO}_{2} \mathrm{CB}$, which implies that the electron injection from excited state of dyes to $\mathrm{TiO}_{2}$ are thermodynamically feasible. However, when the dye is attached to the titania surface and is also in contact with the electrolyte solution a modification of the levels of the LUMO and $\mathrm{TiO}_{2} \mathrm{CB}$ is possible. The redox potential of Spiro-OMeTAD is more positive than the LUMO levels of dyes, which means all dyes can be regenerated by Spiro-OMeTAD. One can find that the introduction of buthoxyphenyl units in donor or more conjugated segment in linker can narrow the $E_{0-0}$ energy gap, which is responsible for the red-shifted absorption spectra of D35 and LEG4 as compared to L1.

\section{2. ssDSCs fabrication and characterization}

The ssDSCs fabrication was carried out according to slightly modified procedure reported before. ${ }^{6,22}$ Fluorine-doped tin-oxide (FTO) coated glass substrates were patterned by etching with zinc powder and $3.5 \mathrm{M}$ hydrochloric acid. A compact $\mathrm{TiO}_{2}$ blocking layer was first deposited onto the surface of a pre-cleaned FTO substrate by spray pyrolysis on a hotplate at $500^{\circ} \mathrm{C}$ using an airbrush. The solution used in the spray pyrolysis was $0.2 \mathrm{M}$ Ti-Isopropoxide, $2 \mathrm{M}$ Acetylacetone in isopropanol and 10 spray cycles were used as standard parameter. Nanoporous $\mathrm{TiO}_{2}$ films were coated on the compact

Table 1. Physical properties of dyes.

\begin{tabular}{lcccc}
\hline & \multicolumn{3}{c}{ HOMO } & LUMO \\
Dye & UV-Vis $(\varepsilon)$ & {$[$ V versus NHE $]$} & {$[$ V versus NHE $]$} & $E_{0-0}[\mathrm{eV}]$ \\
\hline L1 & $483(19000)^{\mathrm{a}}$ & 1.18 & -1.04 & 2.22 \\
D35 & $500(31000)^{\mathrm{a}}$ & $0.97^{20}$ & $-1.21^{20}$ & $2.18^{20}$ \\
LEG4 & $540(49000)^{\mathrm{a}}$ & $0.88^{20}$ & $-1.17^{20}$ & $2.04^{20}$ \\
Z907 & $531(7100)^{\mathrm{b}}$ & $0.65^{21}$ & $-0.98^{21}$ & $1.62^{21}$ \\
\hline
\end{tabular}

${ }^{\mathrm{a}}$ In DCM.

${ }^{\mathrm{b}} \mathrm{In} t$-BuOH:ACN $(1: 1 ; \mathrm{V} / \mathrm{V})$. 
$\mathrm{TiO}_{2}$ layer by screen-printing a colloidal $\mathrm{TiO}_{2}$ paste (Dyesol DSL 18NR-T). The thickness of the film is ca. $2.2 \mu \mathrm{m}$, as measured with a DekTak profilometer. After sintering the $\mathrm{TiO}_{2}$ film on a hotplate at $500^{\circ} \mathrm{C}$ for $30 \mathrm{~min}$, the film was cooled to room temperature and immersed in $0.02 \mathrm{M}$ aqueous $\mathrm{TiCl}_{4}$ at $70^{\circ} \mathrm{C}$ for $30 \mathrm{~min}$. The film was then rinsed by deionized water and then annealed on a hotplate at $500^{\circ} \mathrm{C}$ for $30 \mathrm{~min}$. After cooling to $50^{\circ} \mathrm{C}$, the film was immersed for $2 \mathrm{~h}$ in $0.2 \mathrm{mM}$ dye solution in acetonitrile (AN) and tert-butanol $(\mathrm{t}-\mathrm{BuOH})(1: 1 ; \mathrm{v} / \mathrm{v})$, then the sensitized electrodes were rinsed by ethanol and dried. Subsequently, the chlorobenzene solution containing $150 \mathrm{mM}$ Spiro-OMeTAD, $200 \mathrm{mM} 4$-tert-butylpyridine (TBP) and $20 \mathrm{mM}$ Lithium Bis- (Trifluoromethanesulfonyl)Imide (LiTFSI) was used to form the HTM film by leaving the solution to penetrate into the sensitized electrode for $30 \mathrm{~s}$ and then spin-coating for $3 \mathrm{~s}$ with $1000 \mathrm{rpm}$ and $30 \mathrm{~s}$ with $2000 \mathrm{rpm}$. After HTM deposition, the devices were left in the dark in air overnight. The $200 \mathrm{~nm}$ thick Ag back contact was deposited onto the organic semiconductor by thermal evaporation in a vacuum chamber (Leica EM MED020) with a base pressure of about $10^{-5}$ mbar, to complete the device fabrication. Current-Voltage characteristics were recorded by applying an external potential bias to the cell while recording the generated photocurrent with a Keithley model 2400 digital source meter. The light source was a $300 \mathrm{~W}$ collimated xenon lamp (Newport) calibrated with the light intensity to $100 \mathrm{~mW} / \mathrm{cm}^{2}$ at AM $1.5 \mathrm{G}$ solar light by certified silicon solar cell (Fraunhofer ISE). All ssDSCs samples were illuminated from the glass side with an aperture area of $0.20 \mathrm{~cm}^{2}(0.4 \mathrm{~cm} \times 0.5 \mathrm{~cm})$.

\subsection{Electrochemical measurements}

Electrochemical experiments were performed with a $\mathrm{CH}$ Instruments electrochemical workstation (model $660 \mathrm{~A}$ ) using a conventional three-electrode electrochemical cell. Measurements were obtained with $1 \mathrm{mM}$ solution of dyes in DCM by using $0.1 \mathrm{M}$ Tetrabutylammoniumhexafluorophosphate $\left(\mathrm{TBAPF}_{6}\right)$ as supporting electrolyte. A glassy carbon disk was used as the working electrode, a platinum wire served as a counter electrode, a $\mathrm{Ag} / \mathrm{Ag}^{+}$electrode was utilized as a reference electrode and the scan rate used was $50 \mathrm{mV} / \mathrm{s}$. All redox potentials were calibrated versus a normal hydrogen electrode (NHE) by the addition of ferrocene as an internal standard taking $\mathrm{E}\left(\mathrm{Fc} / \mathrm{Fc}^{+}\right)=630 \mathrm{mV}$ versus NHE. ${ }^{23}$

\subsection{IS measurement}

Acquisition of the IS measurements were carried out with a Frequency Response Analyzer, FRAequipped PGSTAT-30 from Autolab. The amplitude of the AC signal was $25 \mathrm{mV}$ at low applied voltages and $10 \mathrm{mV}$ at high forward bias. The frequency range scanned values between $400 \mathrm{kHz}$ and $0.01 \mathrm{~Hz}$ at the different $V_{\text {app }}$. Illumination was provided by a $1000 \mathrm{~W}$ class-A solar simulator from Newport, filtered at AM1.5 G and with the light intensity adjusted with an NREL-calibrated Si solar cell with a KG-5 filter to 1 sun $\left(100 \mathrm{~mW} / \mathrm{cm}^{2}\right)$.

\section{Results and Discussion}

ssDSCs based on Spiro-OMeTAD as HTM have been fabricated using the four dyes (Z907, L1, D35 and LEG4) as sensitizers to measure their performance in photovoltaic devices. Table 2 collects the photovoltaic properties of fresh ssDSCs based on the different dyes obtained from the current densityvoltage $(J-V)$ curves shown in Fig. 4(a). It is observed that large differences between the two groups of cells, D35-LEG4 and Z907-L1, occur in the photocurrent $\left(J_{\mathrm{sc}}\right)$ and the open circuit voltage $\left(V_{\mathrm{oc}}\right)$. Assuming that the light absorption is proportional to the molar extinction coefficient, i.e., the dye loading and $\mathrm{TiO}_{2}$ thickness are similar for all the dyes, the differences in photocurrent can be explained from the UV-Vis spectra and the molar extinction coefficient of the diverse dyes (Fig. 2 and Table 1), where the molar extinction coefficient value decreases in the direction LEG4 $>$ D35 $>$ L1 > Z907 as the photocurrent is obtained from the $J-V$ curves in Fig. 4.

In order to get further insight into the mechanism governing the performance of the devices and

Table 2. Photovoltaic properties of ssDSCs based on different dyes. ${ }^{\text {a }}$

\begin{tabular}{lcccc}
\hline Dye $^{\text {b }}$ & $J_{\text {SC }}\left[\mathrm{mA} \cdot \mathrm{cm}^{-2}\right]$ & $V_{\mathrm{OC}}[\mathrm{mV}]$ & $\mathrm{ff}$ & $\eta^{\mathrm{c}}[\%]$ \\
\hline L1 & 5.0 & 750 & 0.55 & 2.1 \\
D35 & 7.2 & 905 & 0.64 & 4.2 \\
LEG4 & 7.5 & 915 & 0.67 & 4.6 \\
Z907 & 5.6 & 765 & 0.49 & 2.1 \\
\hline
\end{tabular}

${ }^{\mathrm{a}} 100 \mathrm{~mW} \cdot \mathrm{cm}^{-2}$ light illumination, $0.2 \mathrm{~cm}^{2}$ active area. ${ }^{\mathrm{b}} 2.2 \mu \mathrm{m} \mathrm{TiO}_{2}$ films $\left(0.25 \mathrm{~cm}^{2}\right)$ sensitized in $0.2 \mathrm{mM}$ dye solution in AN:t-BuOH (1:1) for $6 \mathrm{~h}$.

c $150 \mathrm{mM}$ Spiro-OMeTAD, $200 \mathrm{mM}$ TBP and 20 mMLiTFSI in $\mathrm{PhCl}$. 


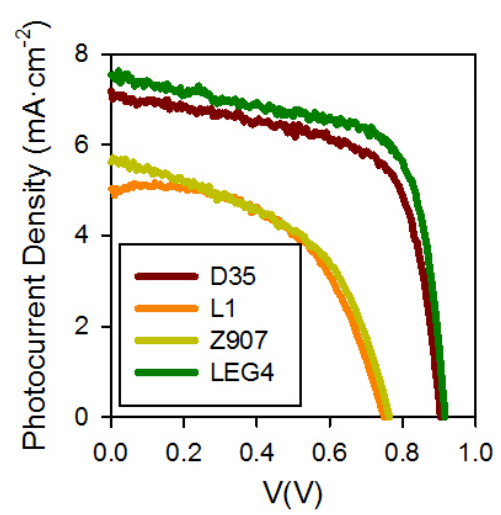

(a)

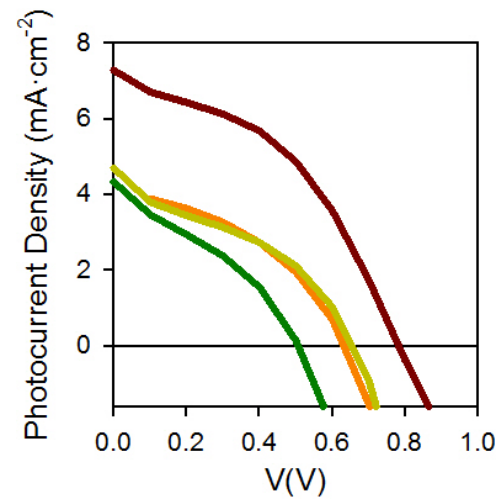

(b)

Fig. 4. $J-V$ curves. (a) Measured as made in KTH laboratory's and (b) after IS measurement.

explain the observed differences in the $V_{\text {oc }}$, IS measurements were performed on the ssDSCs at different bias potentials under one-sun illumination (Fig. 5). These measurements were analyzed using the impedance model developed previously, ${ }^{24-26}$

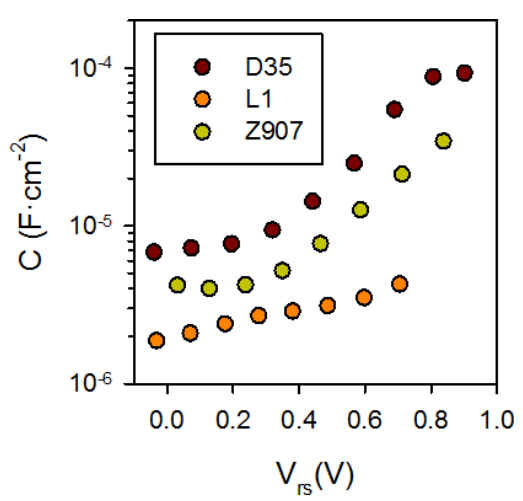

(a)

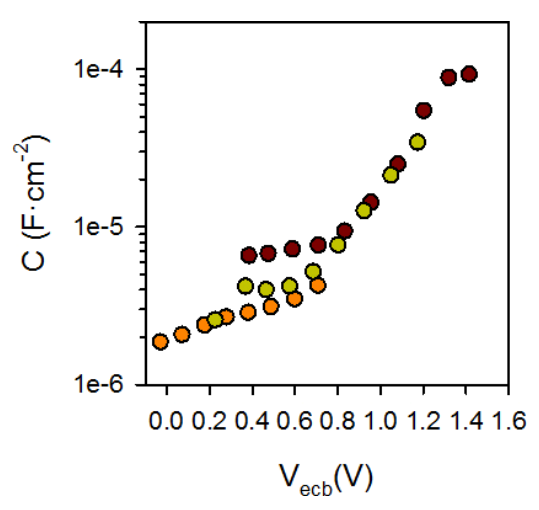

(c) allowing us to isolate the recombination resistance from other resistive contributions in the cell. The aim of IS measurements is to clarify the differences in the cells concerning shifts of the conduction band of $\mathrm{TiO}_{2}$ and in the $V_{\text {oc }}$ values from variations in the

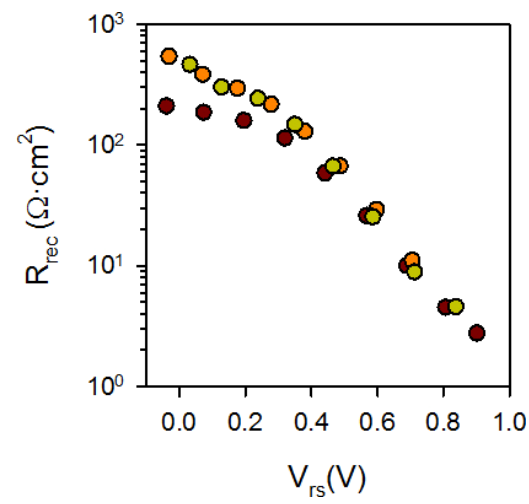

(b)

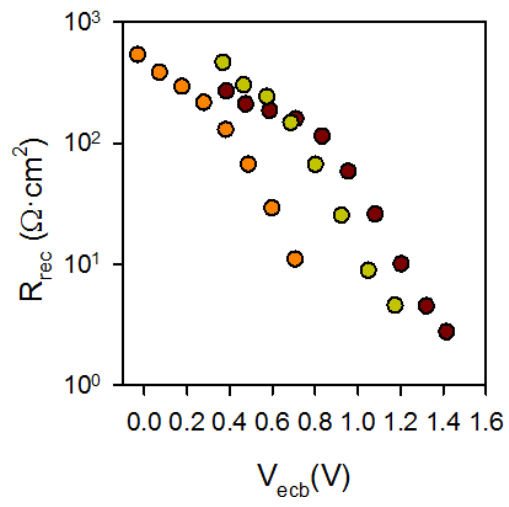

(d)

Fig. 5. (a) Capacitance and (b) recombination resistance, with respect to the Fermi level voltage (removing the effect of series resistance). (c) Capacitance and (d) recombination resistance plotted with respect to the equivalent common conduction band voltage so that the distance between the Fermi level and the conduction band is the same in all cases. 
rate of recombination, ${ }^{27}$ since it has been observed that organic dyes exert a strong influence on the rate of charge transfer resistance. ${ }^{28}$ Related with the charge injection from the dye, with IS it is possible to correlate the position of the conduction band with the charge injection. Lower CB produces higher driving force between the dye excited state and CB of the $\mathrm{TiO}_{2}$ and therefore higher charge injection rate. It was observed in the IS measurements that the collection efficiency for this ssDSCs $(2.2 \mu \mathrm{m}$ thickness of titania film $)$ is not a limitation in its performance, as the electron transport resistance is negligible compared to the recombination resistance.

In order to check the stability of the samples during IS measurements, $J-V$ curves were carried out before and after IS measurement. Good stability has been obtained for D35, L1 and Z907, but a high degradation was observed for LEG4 dye (Fig. 4(b)). Consequently, IS analysis was carried out just on stable devices.

From the analysis of chemical Capacitance versus applied potential (Fig. 5(a)) it is clearly observed that the dye influences the CB level of $\mathrm{TiO}_{2}$, with D35 presenting the lowest conduction band minimum value. Low $\mathrm{CB}$ can enhance the photocurrent as it increases the injection driving force, ${ }^{29}$ for example it is confirmed from the photovoltaic parameters shown in Table 2 and Fig. 4(a) where the short circuit current is $7.2 \mathrm{~mA} / \mathrm{cm}^{2}$, one of the highest charge injection photocurrents, due to the CB shift. On the other hand, the type of dye also greatly influences the recombination process, as seen in the graph of recombination resistance $\left(R_{\text {rec }}\right)$ where all the cells are compared at the equivalent conduction band voltage $\left(V_{\text {ecb }}=V_{F}+q \Delta E_{c}\right)$, where $V_{F}$ is voltage without the effect of the series resistance, see Fig. 5(d). Comparison of samples in terms of $V_{\text {ecb }}$ allows to remove the effect of the different CB position. ${ }^{27,30}$ To perform this comparison, capacitances are just shifted in order to make that all of them overlap, see Fig. 5(c). The same shift is applied to the $R_{\text {rec }}$ in Fig. 5(d). Lower recombination (higher $R_{\text {rec}}$ ) is obtained for D35 due to its structure with bulky moieties in the donor part of the structure (two buthoxyphenyl groups) that act as blocking barrier for electron back recombination, ${ }^{30}$ and the electron donating units on the TPA group, which together with the lower position of the CB increase the total charge collection efficiency and provides the higher photocurrents obtained for D35 (Fig. 4).
These characteristics together with its high extinction coefficient make D35 the ideal dye for ssDSCs.

Z907 dye presents slightly lower recombination resistance than D35 dye, that combined with the lower photocurrent due to its lower molar extinction coefficient produces a smaller $V_{\text {oc }}$ and all together a decrease in the overall efficiency of the cell compared with D35.

For the case of $\mathrm{L} 1$ the situation is different as this dye presents higher molar extinction coefficient than Z907. However, the CB of $\mathrm{TiO}_{2}$ is at a higher energy position, yielding the lower photocurrent obtained because of the lower electron injection efficiency from the dye to the $\mathrm{TiO}_{2}$. As plotted in Fig. 5(b), recombination is again similar to the previous cases despite the larger CB, that is explained by the lack of blocking moieties in the donor part of the dye structure ${ }^{31}$ which generate a strong decrease in the $V_{\text {oc }}$ value. This yields an increased recombination rate as shown by $R_{\text {rec }}$ when represented versus $V_{\text {ecb }}$ in Fig. 5(d). The final result is that L1 has a similar performance than Z907, but for different reasons. In this sense, the impedance analysis gives important clues to analyze the performance of different dyes in ssDSCs. The lower FF for L1 and Z907 could be explained due to the higher recombination in both dyes. ${ }^{25}$

These results state that D35 dye with two butoxyphenyl electron donating units on the TPA, a single linker (thiophene) and high extinction coefficient presents the optimal design to obtain high efficiency solid state DSC compared with standard $\mathrm{Ru}$ dye Z907 and simple organic dye L1, without blocking groups in the donor part of the dye molecule. The origin of the better performance has been shown to be due to both, the higher absorption of light, and the lower recombination rate that offsets the lower $\mathrm{TiO}_{2} \mathrm{CB}$ position.

\section{Conclusions}

Four dyes including three organic dyes and a $\mathrm{Ru}-$ based photosensitizer have been used to investigate the effect of different molecular structures on performance of ssDSCs devices. The extinction coefficient of the molecular dyes combined with the position of the $\mathrm{CB}$ in the semiconductor play a dramatic role in the final cell performance, relatively higher than in conventional sensitized liquid cells as the thickness of the $\mathrm{TiO}_{2}$ layer is limited in solid devices. From the analysis of IS studies, it is found that the different dyes show significant influences on 
$\mathrm{CB}$ of $\mathrm{TiO}_{2}$ and in the recombination resistance as a function of its structure. Specifically, the bulky donor part of the D35 dye shifts the $\mathrm{TiO}_{2}$ conduction band down as compared to L1 and Z907 dye, which is related with higher charge injection that presents D35 dye and together with the red-shifted absorption spectrum, high extinction coefficient and lower charge recombination make the D35 dye the optimum dye for ssDSCs.

\section{Acknowledgments}

This work was financially supported by the Swedish Energy Agency, the Knut and Alice Wallenberg Foundation, National Natural Science Foundation of China (21120102036, 91233201), National Basic Research Program of China (2009CB220009) and China Scholarship Council (CSC). We acknowledge support by a project from Generalitat Valenciana (ISIC/2012/008). H. Tian would like to thank Sillén scholarship for supporting his visiting research in UJI and also thank Rafael Sánchez, Victoria González and others from GDFO for their kind help with this research work.

\section{References}

1. U. Bach, D. Lupo, P. Comte, J. E. Moser, F. Weissortel, J. Salbeck, H. Spreitzer and M. Grätzel, Nature 395, 583 (1998).

2. H. J. Snaith, A. Petrozza, S. Ito, H. Miura and M. Grätzel, Adv. Funct. Mater. 19, 1810 (2009).

3. N. Cai, S.-J. Moon, L. Cevey-Ha, T. Moehl, R. Humphry-Baker, P. Wang, S. M. Zakeeruddin and M. Grätzel, Nano Lett. 11, 1452 (2011).

4. H. Tian, I. Bora, X. Jiang, E. Gabrielsson, K. M. Karlsson, A. Hagfeldt and L. Sun, J. Mater. Chem. 21, 12462 (2011).

5. X. Jiang, K. M. Karlsson, E. Gabrielsson, E. M. J. Johansson, M. Quintana, M. Karlsson, L. Sun, G. Boschloo and A. Hagfeldt, Adv. Funct. Mater. 21, 2944 (2011).

6. L. Yang, B. Xu, D. Bi, H. Tian, G. Boschloo, L. Sun, A. Hagfeldt and E. M. J. Johansson, J. Am. Chem. Soc. 135, 7378 (2013).

7. H. J. Snaith, A. J. Moule, C. Klein, K. Meerholz, R. H. Friend and M. Grätzel, Nano Lett. 7, 3372 (2007).

8. U. Bach, Y. Tachibana, J.-E. Moser, S. A. Haque, J. R. Durrant, M. Grätzel and D. R. Klug, J. Amer. Chem. Soc. 121, 7445 (1999).
9. H. Lee, H. C. Leventis, S.-J. Moon, P. Chen, S. Ito, S. A. Haque, T. Torres, F. Nüesch, T. Geiger, S. M. Zakeeruddin, M. Grätzel and M. K. Nazeeruddin, Adv. Funct. Mater. 19, 2735 (2009).

10. J. A. Chang, J. H. Rhee, S. H. Im, Y. H. Lee, H.-J. Kim, S. I. Seok, M. K. Nazeeruddin and M. Gratzel, Nano Lett. 10, 2609 (2010).

11. T. Fukumoto, T. Moehl, Y. Niwa, M. K. Nazeeruddin, M. Grätzel and L. Etgar, Adv. Energy Mater. 3, 29 (2013).

12. V. González-Pedro, X. Xu, I. Mora-Seró and J. Bisquert, ACS Nano 4, 5783 (2010).

13. M. M. Lee, J. Teuscher, T. Miyasaka, T. N. Murakami and H. J. Snaith, Science 338, 643 (2012).

14. H.-S. Kim, C.-R. Lee, J.-H. Im, K.-B. Lee, T. Moehl, A. Marchioro, S.-J. Moon, R. Humphry-Baker, J.-H. Yum, J. E. Moser, M. Gratzel and N.-G. Park, Sci. Rep. 2, 591 (2012).

15. J. Burschka, N. Pellet, S.-J. Moon, R. HumphryBaker, P. Gao, M. K. Nazeeruddin and M. Gratzel, Nature 499, 316 (2013).

16. M. Liu, M. B. Johnston and H. J. Snaith, Nature 501, 395 (2013).

17. J. H. Heo, S. H. Im, J. H. Noh, T. N. Mandal, C.-S. Lim, J. A. Chang, Y. H. Lee, H.-J. Kim, A. Sarkar, K. NazeeruddinMd, M. Gratzel and S. I. Seok, Nat. Photon 7, 486 (2013).

18. H.-S. Kim, I. Mora-Sero, V. Gonzalez-Pedro, F. Fabregat-Santiago, E. J. Juarez-Perez, N.-G. Park and J. Bisquert, Nat. Commun. 4, 2242 (2013).

19. J. Burschka, A. Dualeh, F. Kessler, E. Baranoff, N.-L. Cevey-Ha, C. Yi, M. K. Nazeeruddin and M. Grätzel, J. Am. Chem. Soc. 133, 18042 (2011).

20. E. Gabrielsson, H. Ellis, S. Feldt, H. Tian, G. Boschloo, A. Hagfeldt and L. Sun, Adv. Energy Mater. (2013), doi: 10.1002/aenm.201300367.

21. L. Giribabu, V. K. Singh, C. Vijay Kumar, Y. Soujanya, V. Gopal Reddy and P. Yella Reddy, Adv. Optoelectron. 2011, 8 (2011).

22. B. Xu, H. Tian, D. Bi, E. Gabrielsson, E. M. J. Johansson, G. Boschloo, A. Hagfeldt and L. Sun, J. Mater. Chem. A. 1, 14467 (2013).

23. D. P. Hagberg, T. Edvinsson, T. Marinado, G. Boschloo, A. Hagfeldt and L. Sun, Chem. Commun. 21, 2245 (2006).

24. J. Bisquert, J. Phy. Chem. B 106, 325 (2001).

25. F. Fabregat-Santiago, J. Bisquert, E. Palomares, L. Otero, D. Kuang, S. M. Zakeeruddin and M. Grätzel, J. Phys. Chem. C 111, 6550 (2007).

26. E. M. Barea, V. Gonzalez-Pedro, T. Ripollés-Sanchis, H.-P. Wu, L.-L. Li, C.-Y. Yeh, E. W.-G. Diau and J. Bisquert, J. Phys. Chem. C 115, 10898 (2011). 
27. S. R. Raga, E. M. Barea and F. Fabregat-Santiago, Phys. Chem. Lett. 3, 1629 (2012).

28. B. C. O'Regan and J. R. Durrant, Acc. Chem. Res. 42, 1799 (2009).

29. E. M. Barea, C. Zafer, B. Gultekin, B. Aydin, S. Koyuncu, S. Icli, F. F. Santiago and J. Bisquert, $J$. Phys. Chem. C. 114, 19840 (2010).
30. F. Fabregat-Santiago, G. Garcia-Belmonte, I. MoraSero and J. Bisquert, Phys. Chem. Chemi. Phys. 13, 9083 (2011).

31. E. M. Barea and J. Bisquert, Langmuir 29, 8773 (2013). 